\title{
DESIGN WITH SADSF METHOD AND ANALYSES OF ELASTIC PROPERTIES OF TORSION-LOADED STRUCTURES BASED ON DOUBLE-TEE SECTIONS
}

\author{
IRENEUSZ MARKIEWICZ \\ Kielce University of Technology, Faculty of Mechatronics and Mechanical Engineering, Kielce, Poland \\ e-mail: ireneusz.markiewicz@tu.kielce.pl
}

\begin{abstract}
The paper presents results of the preliminary strength design using the method of statically admissible discontinuous stress fields (SADSF) of two new and interesting thin-walled structures based on double-tee sections. Although these constructions are intended to carry torsion moment loads, all their surfaces are accessible from outside. The paper is completed with the selected results of linearly-elastic FEM analyses of the presented solutions. They show surprisingly good strength properties and significantly higher load-carrying capacity comparing to structures designed in an intuitive way. The objectives of the paper, among other things, are as follows: popularization of the SADSF method, presentation of its new solutions and confirmation of practical usefulness in the design of thin-walled structures.
\end{abstract}

Keywords: thin-walled structures, design, limit analysis, shape and topology optimization, SADSF method

\section{Introduction}

Due to their properties, thin-walled structures are commonly applied, and the methods concerning strength design are intensively developed and represent the centre of interests of scientists.

The main difficulty encountered in designing these systems is that even apparently minor changes in their constructional details or changes in boundary conditions may cause large and non-local changes in stress and deformation fields (e.g. Bodaszewski, 2013). For this reason, the design methods based on intuitive or iterative improvements, including contemporary, quickly developed methods of topology optimization (Bendsoe and Sigmund, 2003; Huang and Xie, 2010; Mróz and Bojczuk, 2003), must be applied with great caution.

The paper presents a method which does not make use of iterative procedures and uses statically admissible, discontinuous stress fields, and is justified by the lower-bound theorem of limit analysis and is called SADSF (Szczepiński, 1968; Dietrich et al., 1970; Frąckiewicz et al., 1986; Szczepiński and Szlagowski, 1990; Szlagowski, 1990; Zowczak, 2004; Bodaszewski and Szczepiński, 2005; Bodaszewski, 2013).

A manner of formulating the design problem typical for this method is presented in Fig. 1a (Bodaszewski, 2013). The only data is: limit load at the boundary $S_{p}$ reduced to two pairs of forces $P h$, geometry of this part of the boundary (dimensions: $L, h, e$ ), and the yield point of the structure material $\sigma_{Y}$.

One should notice that analogical formulations which use only the data on boundary conditions, appear during designing each new structure.

In order to solve the presented problem using the SADSF method, it is necessary to construct a statically admissible, discontinuous stress field, which would satisfy the given boundary conditions and specify the structure configuration, i.e. number, spatial positioning and system of mutual connections of component elements as well as determine the shape and dimensions of these elements. Therefore, this field will completely define the sought for structure. 
Statically admissible stress fields satisfy only the equilibrium equations and static boundary conditions and do not infringe the assumed yield condition. The SADSF method assumes making use of discontinuous fields wherein lines of discontinuity are sections of straight lines which cause that stress states are homogenous within any area. During construction of the fields, one strives for a situation where the yield condition is achieved within all or at least as many areas as possible. Moreover, it is assumed that the constructed spatial fields consist of plane fields fragments.

Basic difficulty during construction of such fields is that for arbitrarily selected networks of lines of stress discontinuities (division to homogenous areas), there is usually no solution. At the beginning, even the arrangement of conditions that need to be set is unknown, and one must notice that these are non-linear equations and inequalities which include singularities, and usually large number of variables (Bodaszewski, 2004, 2005). This causes that direct approaches in the SADSF method cannot be applied.

These difficulties are bypassed within the scope of the application version of the SADSF method, created especially for engineers. An engineer does not have to solve any new statically admissible stress fields. He or she uses a set of ready-made library fields (Fig. 1b) of a low level of complexity, which are delivered together with the software. The fields are assembled into more complex ones in such a way that the assumed boundary conditions and equilibrium conditions are satisfied.

At the moment, the most advanced package of the application version is SADSFaM (Bodaszewski, 2013). It is based on the concept of a multi-level idea of constructing complex fields and allows simple design of even the most complex thin-walled structures consisting of flat elements.

In this idea, the library fields of the package, presented in Fig. 1b, represent the fields of level I and are oriented at designing thin-walled structures. Flat and spatial solutions of average complexity made of them are the fields of level II. Fields such as these are constructed and catalogued by a designer in order to use them as fragments - usually repeating ones - of even more complex fields, which represent solutions of the considered problems (e.g. Fig. 1c and 1d) and are classified as fields of level III or higher. A fragment of the existing library of fields of level II is presented in Fig. 1e (Bodaszewski, 2013).

The satisfaction of the criterion of yield condition utilized in the SADSF method, within as many areas as possible, does not lead to unequivocal solutions. This plurality of possible solutions is useful from the standpoint of the designer because it allows satisfying additional conditions, for example resulting from preliminary design assumptions or related to the simplicity of execution, strength, etc. The number of solutions that may be constructed is limited to these which can be assembled based on the fields available in the library.

Two new and original solutions of the problem, Fig. 1a, developed within the scope of the paper are presented in Figs. 1c and 1d. They may be used, for example, as cross-beams of load-carrying frames of vehicles. All their surfaces are accessible from outside and, despite this, the structures exhibit high rigidity to torsion.

The constructed statically admissible fields require application of systems of additional elements in the central part of the structures. In the solution from Fig. 1c, these elements have the shape of letter X (solution denoted as $Z 2 x 93$ ) while in the solution from Fig. 1d (the solution denoted as $Z 2 x 94$ ) they look like a rectangular pipe.

In each library field, one assumes realization of a plane state of stress, the result of which is that the membrane state should be able to transfer the assumed load. Therefore, structures may successfully utilize the load-carrying properties of materials used to build them. If any of their elements is removed or not connected with welds at the edges, where the fields show non-zero interactions, the load-carrying capacity would be reduced by one order. This is a cardinal error.

The designed structures are free of cardinal errors, therefore, one may expect their high quality level. 
(a)

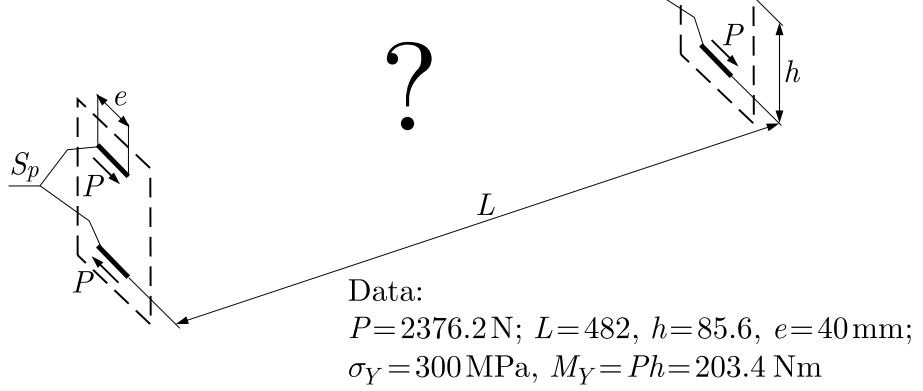

(c)
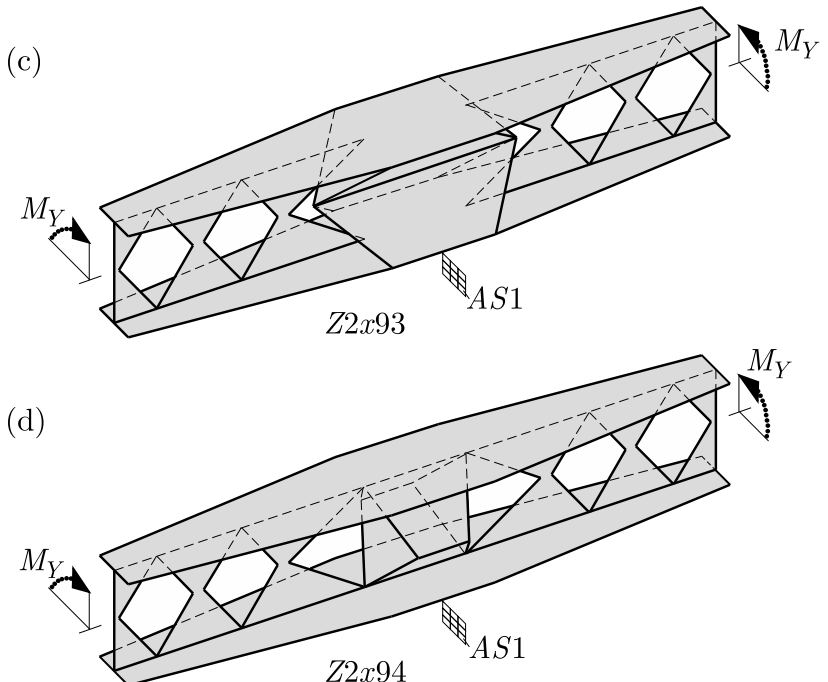

(b)

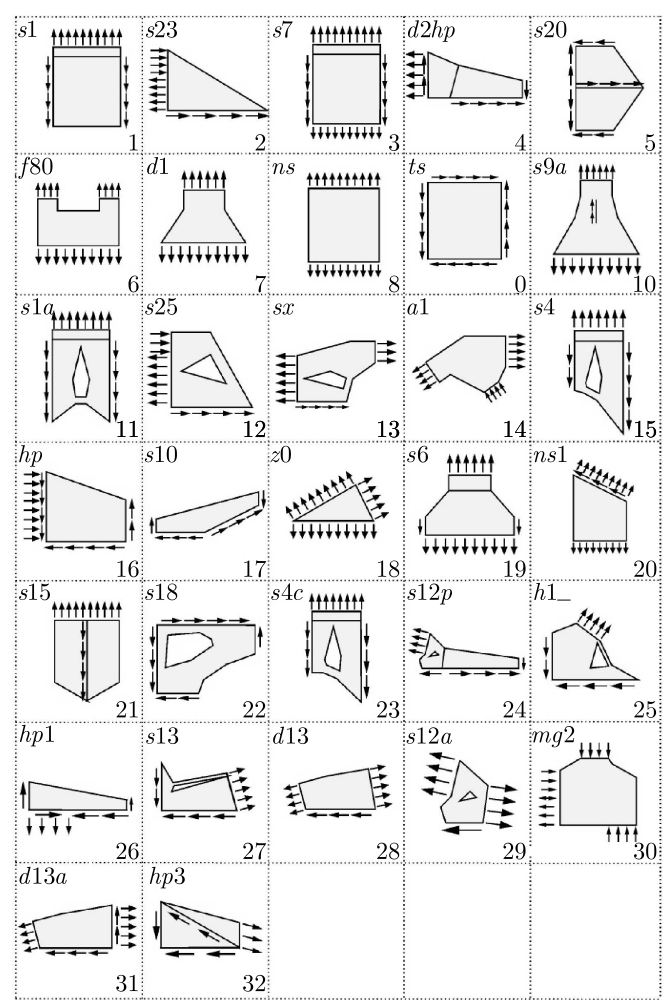

(e)

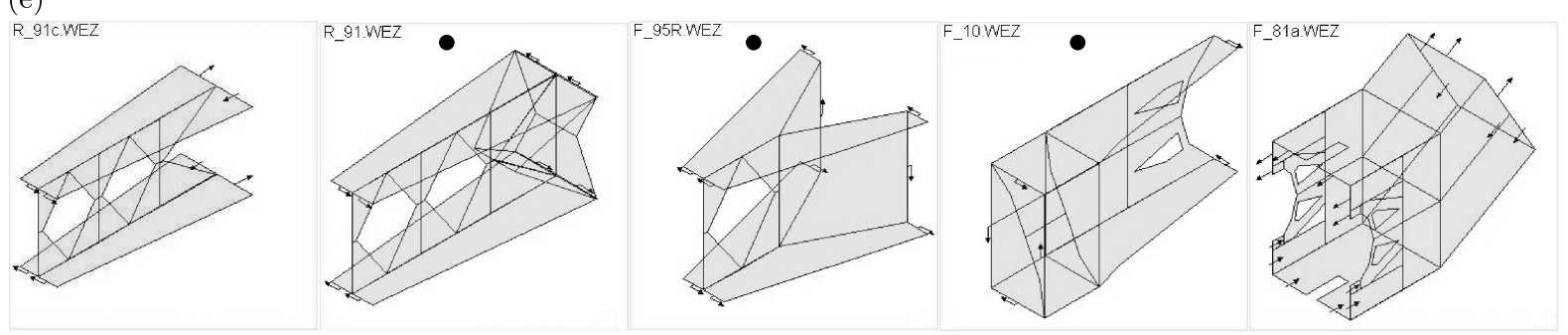

Fig. 1. Formulation and solutions of the complex field construction problem: (a) graphical illustration of the design problem formulation; (b) library of ready-made particular solutions from SADSFaM software package, without stress discontinuity lines; (c), (d) solutions: contours of statically admissible stress fields which specify the sought for structures; (e) fragment of an existing library of a designer, without stress discontinuity lines

These properties are proven by e.g. elastic FEM analyses of one of the developed solutions included in the paper. They also show possible benefits emerging from utilization of the SADSF method during designing the thin-walled structures. These properties are expected also based on the conclusions presented, among other things, in the papers by Markiewicz (2007, 2013).

\section{Basic information concerning SADSFaM package (Bodaszewski, 2013)}

Examples of four types of library fields of SADSFaM package, which have been used to design the solutions presented in the paper, are shown in Figs. 2a-d. As one can see, each of them 
satisfies different but relatively simple boundary conditions and is described within own local coordinate system $\{a\}$ and has individual designation (here: $H p, T s, N s 1, s 1$ ).

(a)

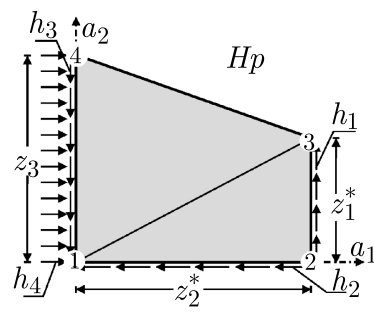

(b)

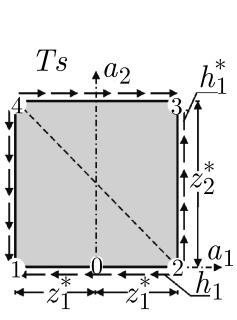

(c)

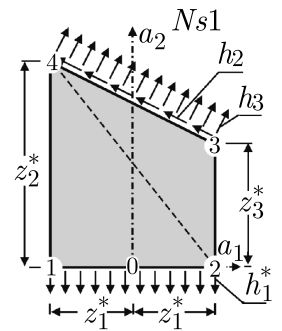

(d)

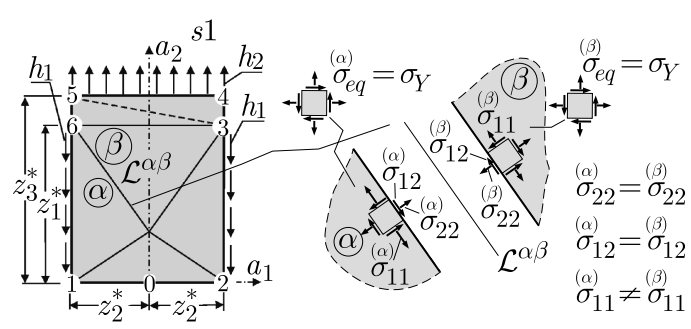

Fig. 2. The library fields of the SADSFaM package, which are used in the solutions presented in the paper and the interactions along the line of stress discontinuity $\mathcal{L}^{\alpha \beta}$ in the system related to this line

The field loads are described by external stress parameters $h_{i}$, which are coordinates of stress vectors applied at the edges. Location of loaded edges is described by external geometrical parameters $z_{i}$, which have the form of linear dimensions.

In the case of each of such fields, equilibrium equations for each stress discontinuity line must be met. An example of such a line $\mathcal{L}^{\alpha \beta}$ separating two different homogenous stress areas $\alpha$ and $\beta$, together with equilibrium conditions, is presented in Fig. 2e. As it can be seen, the coordinates $\stackrel{(\alpha)}{\sigma_{22}}, \stackrel{(\beta)}{\sigma_{22}}$ and $\stackrel{(\alpha)}{\sigma}_{12}, \stackrel{(\beta)}{\sigma}_{12}$ must be equal. Only the coordinates $\stackrel{(\alpha)}{\sigma}_{11}$ and $\stackrel{(\beta)}{\sigma}_{11}$ can differ (if these were equal, the states of stress in both regions would be identical as well).

Satisfaction of these conditions on each of the field discontinuity line results in that its global equilibrium equations must be identically satisfied. Therefore, not all parameters $h_{i}$ and $z_{i}$ can be assumed as independent. Figures 2a-d show parameters, identified with asterisks, that are assumed as independent, and their values may be given in the package.

As mentioned before, while assembling the library fields, the conditions of equilibrium at the connecting edges must be satisfied. These conditions are called joining conditions and include:

- geometrical conditions, i.e. overlapping conditions of common border segments;

- static conditions, i.e. conditions concerning satisfaction of equality of mutual interactions on these segments.

Each of the library fields in the complex solution is given its unique index $s$ (inscribed in quadrilateral rims), and thickness of its element is denoted as $\delta^{(s)}$.

The package SADSFaM makes use of Treska's yield criterion, so the stress coordinates given on the boundaries of fields are calculated with reference to: $k=\sigma_{Y} / 2=150 \mathrm{MPa}$.

\section{Construction of statically admissible stress fields}

While designing the presented solutions, it is assumed that they will show symmetry of shape and antisymmetry of internal forces with reference to three antisymmetry planes of the applied load, which are denoted as $A S 1, A S 2$ and $A S 3$ (Figs. 3 and 4).

The method of constructing a statically admissible field in the half of the solution $Z 2 x 93$ (Fig. 1c), which is denoted as $R_{-}$93, is presented in Fig. 3.

Two fields of level II of complexity, which are presented in Figs. 3a and 3b, are used. Thin arrows in the figures show the resultants of interactions between the component fields and thick arrows show the resultants of external reactions. Component numbers of the library fields are also given together with the fields names in the tables next to these numbers.

The first field, denoted as Ad93-94 adjoins the boundaries $S_{p}$, satisfies boundary conditions given therein and determines the shape and dimensions of flanges and web (Fig. 3a). This is a 

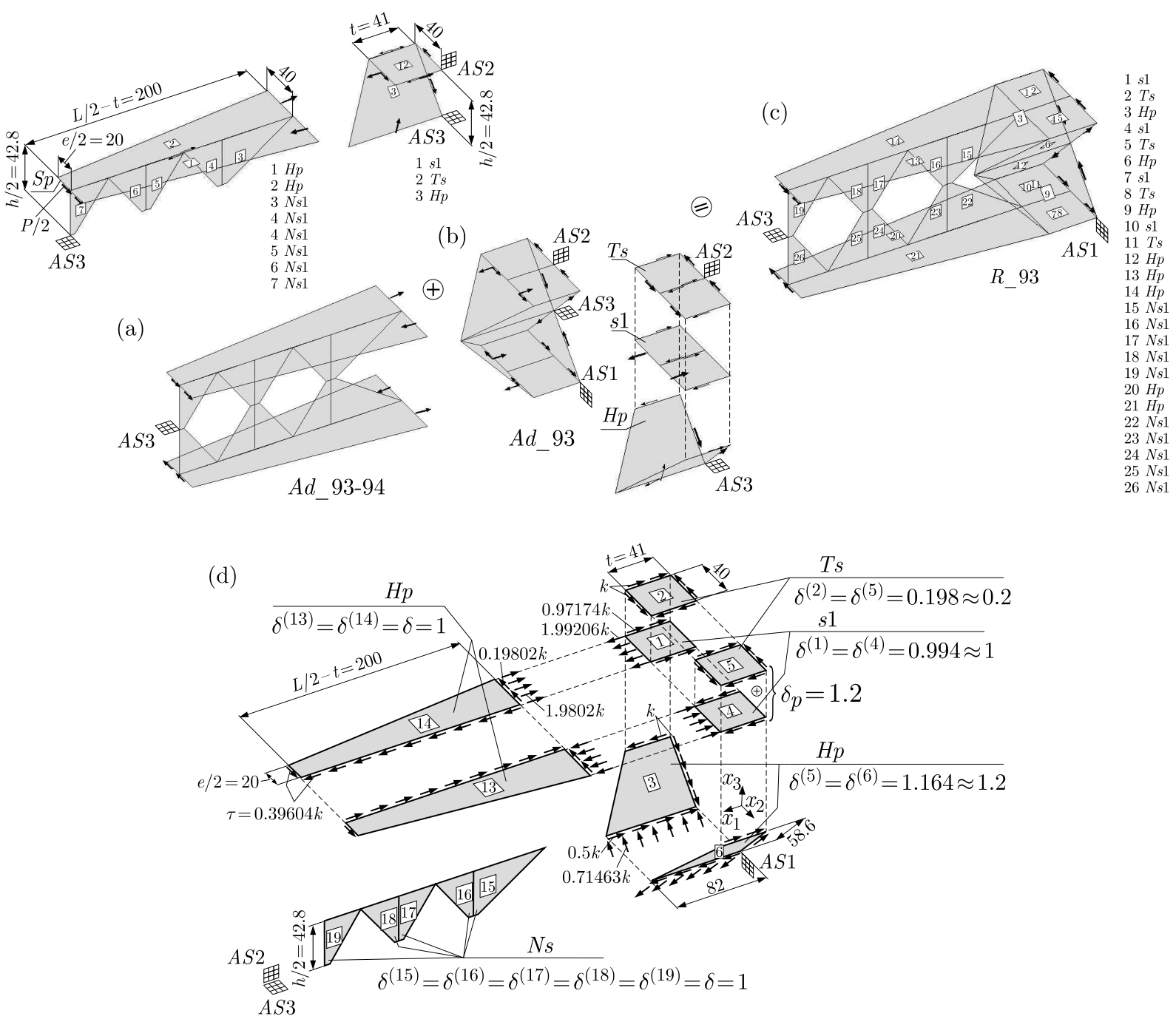

Fig. 3. Construction of the field in the antisymmetric half of the solution $Z 2 x 93$ from Fig. 1c: (a) field $A d \_93-4$, which determines shape and dimensions of flanges and web within the area adjacent to $S_{p}$; (b) field $A d \_93$, which determines shape and dimensions of flanges within the area adjacent to $A S 1$ and additional skew elements; (c) field $R \_93$, which represents half of the solution $Z 2 x 93$; (d) position of the library fields components, interactions between them and thickness of their elements in the top half of the field $R \_93$

modified, from the standpoint of satisfaction of parameters adopted at these boundaries, existing solution $R_{-} 91$ c (Fig. 1e). In the case of the solution concerning this field, it is enough to enter data only for its antisymmetric half, which is presented in the top part of the drawing and then the copy it using one of the package functionalities. This half consists of two fields $H p$ in the flanges and five non-loaded fields $N s 1$ in the web. As one can see, it is assumed that the web does not transfer any loads and it is provided only to "support" the geometry. Arrangement of holes in this element and parameters of fields $N s 1$ can be adopted relatively arbitrarily.

The second field of level II, which determines the shape and dimension of the structure within the space of introduced additional elements, is denoted as $A d \_93$. In order to derive it, it is only necessary to solve and then copy its antisymmetric quarter, which is presented in the top part of Fig. 3b. It consists of only three fields: $s 1, T s$ and $H p$.

The assumed method of load transfer through the component fields is shown in the bottom part of Fig. 1b, wherein the paired library fields from the top half of the solutions (in spaced form) together with interactions between them are presented. 
By combining the fields $A d 93-94$ and $A d \_93$, one gets the statically admissible field $R \_93$, in the half of the solution $Z 2 x 93$, which is presented in Fig. 3c. This field is also qualified as the field of level II of complexity.

The discussed Figs. 3a-c are mostly a sheet of screen copies provided by the SADSFaM package, complemented by selected dimensions and additional denotations. These figures, however, do not include most of the numerical data. That is why, with the use of a graphic software, one carries out additional Fig. 3d which presents the positioning of component fields in the antisymmetric half of the fields from Fig. 3c (in spaced form). Dimensions and interaction values given in this figure may be easily assigned to external parameters of the applied library fields from Fig. 2. Transformation matrices, which specify the position of library fields in space, are not discussed in this paper.

As one may see, normal interactions from the fields $H p(s=13,14)$ are taken over by the fields $s 1(s=1,3)$, and tangent interactions by the fields $T s(s=2,4)$, which perform pure shear. The fields $H p$ and $T s$ are located one above another and represent together an extension of flanges within the area where additional skew elements are introduced.

Balancing the tangent interactions at the external edges of the fields $T s(s=2,4)$ and $s 1$ $(s=1,3)$ necessitate introduction of additional, skew positioned, fields $H p(s=5,6)$. These fields have different values of external parameters comparing to the fields $H p$ in the flanges.

Independent geometrical parameters of the component fields result from the given dimensions: $L, h, e$ and the adopted dimension $t$ (Fig. 3) as well as geometrical conditions of joining.

One must notice that the tangent stress $\tau$ applied at the edges of the fields $H p(s=13,14)$ adjacent to $S_{p}$ is not given while entering data of these fields (it is only specified after introduction of independent external parameters). After its determination, it is possible to determine the thickness of elements of the fields $H p$ using the formula

$$
M_{Y}=\tau \delta e h
$$

Thicknesses of elements of the remaining fields are determined using the static joining conditions. For example, at the common edge of the fields $H p(s=14), s 1(s=1)$ and $T s(s=2)$ such conditions have the following form

$$
1.980 k \delta^{(14)}=1.992 k \delta^{(1)} \quad 0.198 k \delta^{(14)}=1.0 k \delta^{(2)}
$$

In the SADSFaM package, these conditions are arranged and solved automatically. The obtained thicknesses are given in Fig. 3d.

The total thickness of the flange in the area with additional skew elements equals the sum of thicknesses of elements of the fields Ts and $s 1: \delta_{p}=\delta^{(3)}+\delta^{(4)}$ - layer superimposition of these fields has not been performed (Szlagowski, 1990; Bodaszewski, 2004, 2005; Bodaszewski and Szczepiński, 2005).

Only the thickness of elements of the not loaded web may be assumed arbitrarily. It is assumed that it is the same as the thickness of flanges and equals $\delta$.

The second of the complex fields, which is constructed for the half of the solution $Z 2 x 94$ and denoted as $R \_94$, is presented in Fig. 4c. The presentation method is similar to the previous example.

This solution is derived by combining the already discussed field $A d \_93-94$ (Fig. 4a) with the field $A d \_94$, which is presented in Fig. 4b.

As one may see, the fields $s 1(s=1,5)$ play the same role here as in the previous solution (Fig. 4d). One adopted reverse directions of interactions in the fields $T s(s=2,6)$, therefore, it is possible to zero the total interactions on the free edges of the external flanges.

Tangent interactions from the fields $H p(s=17,18)$ and $T s(s=2,6)$ in this case sum up and are balanced by tangent interactions from additionally introduced fields $s 1(s=3,7)$. 


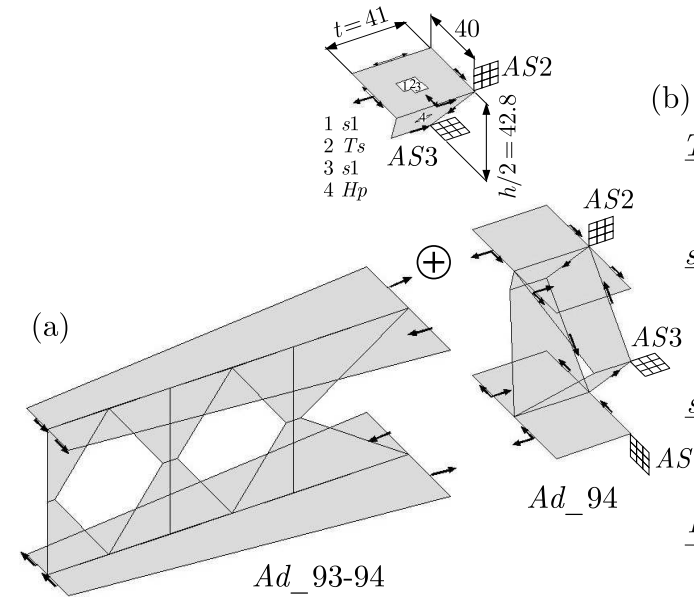

(b)
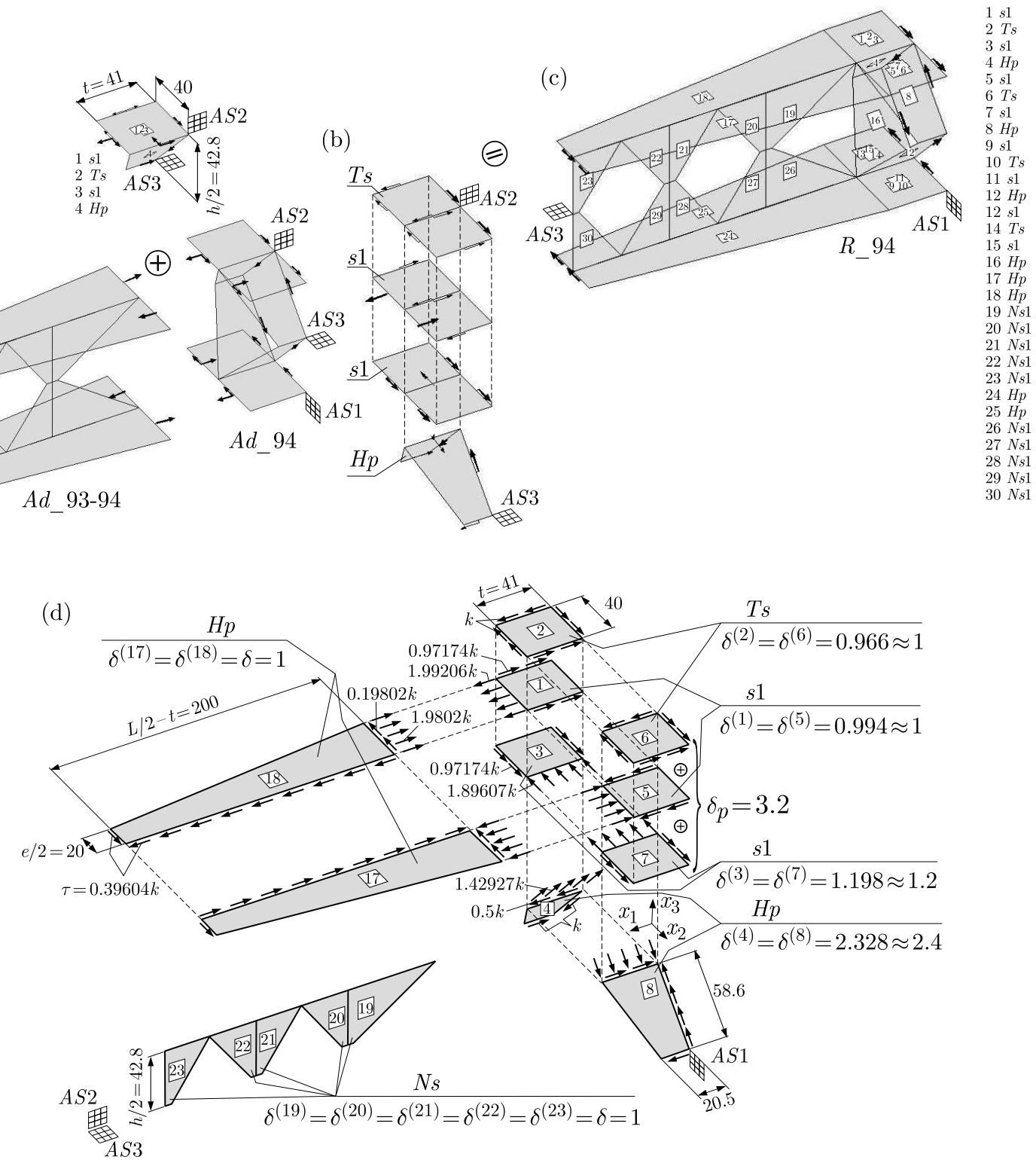

Fig. 4. Construction of the field in the antisymmetric half of the solution $Z 2 x 94$ from Fig. 1d:

(a) field $A d \_93-4$; (b) field $A d \_94$, which determines the shape and dimensions of additional skew elements and flanges in the area adjacent to $A S 1$; (c) field $R_{-} 94$, which represents a half of the solution to the problem $Z 2 x 94$; (d) position of the library fields components, interactions between them and thickness of their elements in the top half of the field $R \_94$

Normal interactions from these additional fields, which act in the web plane, are balanced by normal interactions from the skew located fields $H p(s=4,8)$.

Complete solutions of the problem from Fig. 1a are derived by combining two fields $R \_93$ and two fields $R_{-} \_94$, as shown in Figs. 5a and 5b. As one may see, the joining conditions, due to symmetry of fields geometry and antisymmetry of internal forces, are satisfied identically.

The solutions $R \_93$ and $R \_94$ expand the designer library. Exemplary fields of such a library, which includes hundreds solutions derived by Bodaszewski (2013), are presented in Fig. 1e. The dots denote these fields which can be used to solve the problem from Fig. 1a.

The derived solutions $Z 2 x 93$ and $Z 2 x 94$ are, however, not too complicated fields. Based on the fields in the designer library, solutions of the complete load-carrying frames of vehicles are designed by connecting and copying them (Bodaszewski, 2013). The solutions presented in the paper may be used as cross-beams in these frames. 
Interactions at the edges of the component fields show that the presented structures consist of elements bent in their planes. They will, therefore, form bending axes, and stresses will increment together with an increase in the distance from these axes. Obtaining a balanced elastic equivalent stress in these elements is thus not possible. It is worth to remember when analysing FEM results.

(a)

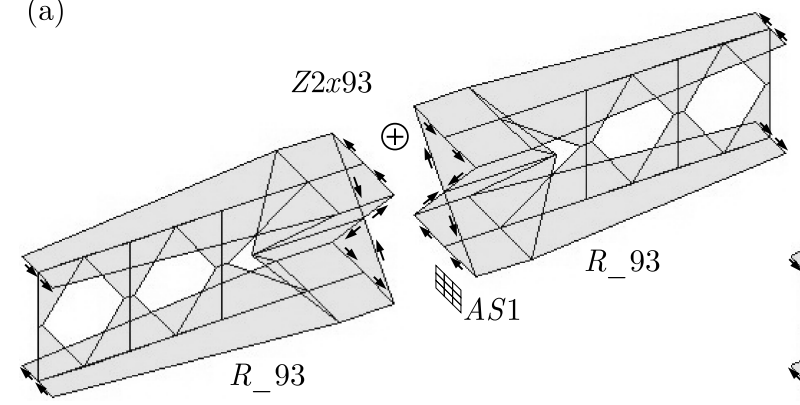

(b)

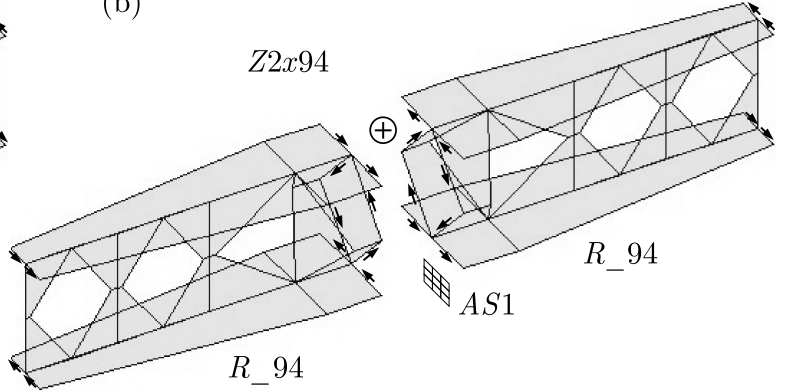

Fig. 5. Composing the derived complex fields $R \_93$ and $R \_94$ into complete solutions of the problem from Fig. 1a

One must also notice that in the planes denoted in Fig. 5a and 5b as $A S 1$, there are no reactions towards perpendicular direction, which would generate a bimoment. In both cases, the bimoment increases together with receding from the extreme sections, however, only to the point of occurrence of additional skew elements. Then it decreases, and in the AS1 section it equals zero. One must mention that near the sections with the greatest bimoment, there are the greatest stress concentrations within the elastic range.

\section{Properties of structures designed using the SADSF method}

The thin-walled structures derived using the SADSF method have structure configurations adapted to the applied load and are free of cardinal errors. However, the method itself is an approximate one and assumes that, among other things, using the rigid-ideally plastic model of material only considers the limit state of the structure corresponding to the beginning of its collapse and assumes maintenance of the membrane state until it is reached. That is why, studies of actual properties of the designed systems at stages and under working conditions, which the SADSF method does not include (elastic, elastic-plastic or time-variable loads), have been carried out since the beginning of its existence (e.g., Szczepiński, 1968; Dietrich et al., 1970; Kapkowski and Stupnicki, 1973; Frąckiewicz et al., 1986; Szczepiński and Szlagowski, 1990; Szlagowski, 1990; Bodaszewski, 1994; Zowczak, 2004; Markiewicz, 2007).

Good or very good properties at these stages proved under these research, require conformation and examination of many case studies so that their results would be representative for a considered class of thin-walled structures. Therefore, within the recent years, a wide range research program has been initiated, which covered (Markiewicz, 2013):

- within the range of elastic-plastic deformations - examinations of yield zones development using thermovision and actual mechanisms of collapse and paths of equilibrium within the whole range of the applied loads;

- within the elastic range - analysis of distribution of the equivalent stress fields using FEM;

- at time-variable loads - estimated fatigue strength using the local strain-life method.

Research within the elastic-plastic range showed, among other things, the same limit load capacities of their components, yielding of significant segments of volumes at the moment of collapse and maintenance of membrane state domination for loads slightly less than the actual 
limit load. The collapse itself was however always caused by great deformation changes of the geometry, and in each case the actual limit load was greater than the one assumed during design.

A few dozen thin-walled structures designed using the SADSF method were subjected to FEM analyses within the elastic range, which was usually the operational one. Small deformations and domination of membrane states, almost accurate equalization of equivalent stress along free borders and relatively low stress concentrations were the rule in that case. It was also found that the designed systems had strength properties far more better comparing to structures designed using intuitive methods.

Results of the linearly-elastic FEM analyses of the structure $Z 2 x 94$ present hereinafter complement the results given in the aforementioned monograph and confirm the mentioned rules within full extent.

Very good properties have been revealed while estimating fatigue strength. It turns out that thin-walled structures designed using the SADSF method have a fatigue life longer by few orders comparing to structures designed using traditional methods, and fatigue cracks can initiate almost simultaneously at various locations.

Based on the performed studies, one may expect good load-carrying properties of the structures. Moreover, the case with poor strength conditions has not been reported so far.

Structures like these can be accepted straight away and in very rare cases further improved. Improvements should apply to geometrical parameters (shape and dimensions of elements) the change of which does not significantly affect the considered class of structures.

\section{FEM analysis results}

To show the quality of the designed structures within the linearly-elastic range, one presents distribution of equivalent stresses derived by means of FEM for the structure model denoted as $Z 2 x 94$. These distributions were compared to distributions specified for a model of "regular" double-tee section which is not designed using SADSF method and the structure of which is not adapted to the torsion moment load. This model includes thus a cardinal error.

During the analyses, the shell model is used, which is approximated and does not allow analysing local three-dimensional states formed within areas of component elements connections. However, it still shows the scale of possible changes in load-carrying properties.

Structural diagrams of both models and the boundary conditions adopted during calculations are presented in Figs. 6a and 7a.

The shape and dimensions of the model from Fig. 6a accurately corresponds to the solution derived from SADSF. The value of the force $F$ is selected so that the value of torsion moment load equals to the half of the moment assumed in the design $M_{Y}$. Taking the accepted yield point value: $\sigma_{Y}=300 \mathrm{MPa}$ and assuming that, in the elastic state of stress, there exists an ideally equalized state of stress, one should obtain the equivalent stress value at each point of the analysed structure equal to: $\sigma_{e q}=150 \mathrm{MPa}$.

The parameters of regular double-tee section model are adopted so that its weight approximately corresponds to the weight of the model designed in SADSF. Thickness of all elements is $1.2 \mathrm{~mm}$, and width of flanges is fixed and equal to the greatest width derived from statically admissible fields. This change forced minor widening of the membranes $p 1$ and $p 2$.

The boundary conditions adopted for this model are the same as in the model $Z 2 x 94$. Only the value of load is assumed to be 16.5-times less so that the level of the greatest equivalent stresses in both models is approximately the same.

The derived distributions of equivalent stresses for the model $Z 2 x 94$ are presented in Fig. 6 . Figure $6 \mathrm{~b}$ shows the distribution of total equivalent stress (membrane and bending), however, Fig. 6c shows a distribution from the component membrane state to the bending state in Fig. 6d. 

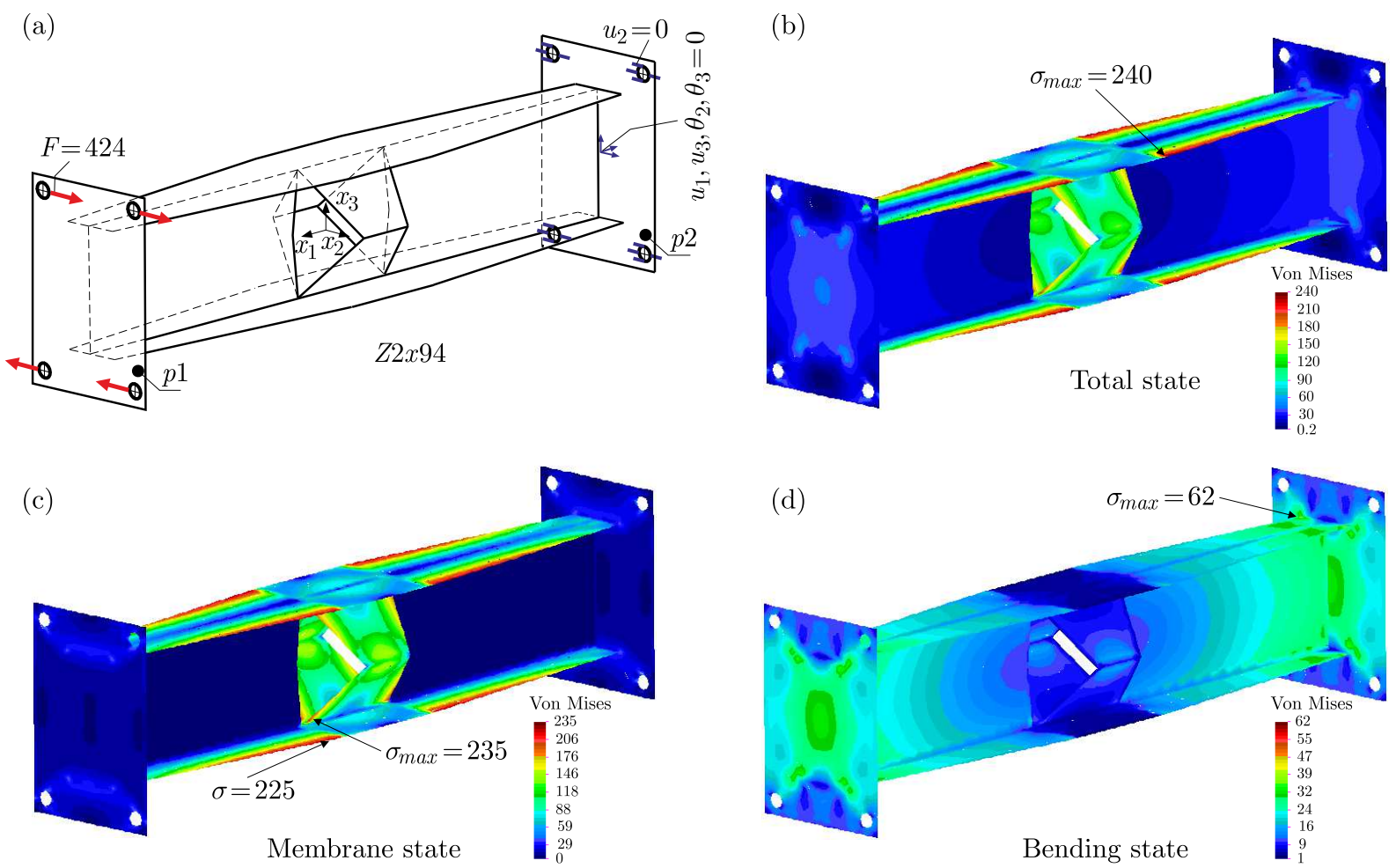

Fig. 6. The adopted boundary conditions and distribution of equivalent stresses calculated according to Huber-Mises-Hencky criterion for the model $Z 2 x 94$

Based on the results, one may determine that:

- domination of the membrane state: the derived value of the greatest equivalent stress from the bending state reaches ca. $26 \%$ of the greatest value derived for the membrane state $(62 / 235 \approx 0.26)$, and the distribution of total stress corresponds approximately to the distribution of the membrane state (Figs. 6b and 6c);

- presence of minor local stress concentrations at various structure locations (e.g. indicated by arrows, where: $\sigma_{e q}=235 \mathrm{MPa}, \sigma_{e q}=225 \mathrm{MPa}$ ), however, values in these locations are similar;

- relatively good equalization of the equivalent stresses from the membrane state along the free edges of flanges;

- occurrence of the greatest total equivalent stresses and from the membrane state on the flange edges near the sections with the greatest bimoment;

- lack of load transfer in the membrane state through the web, wherein zero stress condition is adopted in the design (Fig. 4);

- occurrence of the greatest stresses from the bending state near the extreme membranes, which confirms that a small bimoment is introduced into these elements.

Rigidity of this model is: $\kappa=M / \phi \approx 318 \mathrm{Nm} / \mathrm{deg}$; where: $M$ is the applied torsional moment value, $\phi$ - angle of rotation of the upper border of the membrane $p 1$ (Fig. 6a) calculated based on displacements of its extreme nodes.

In the case of the regular model of double-tee section (Fig. 7), it is found that:

- it is necessary to reduce the applied forces by 16.5-times in order to derive the level of the greatest equivalent stress as in the case of the structure designed using the SADSF method;

- load is transferred mostly through the bending state: value of the greatest equivalent stress from the bending state is ca. 2.4-times greater than the greatest value derived for 

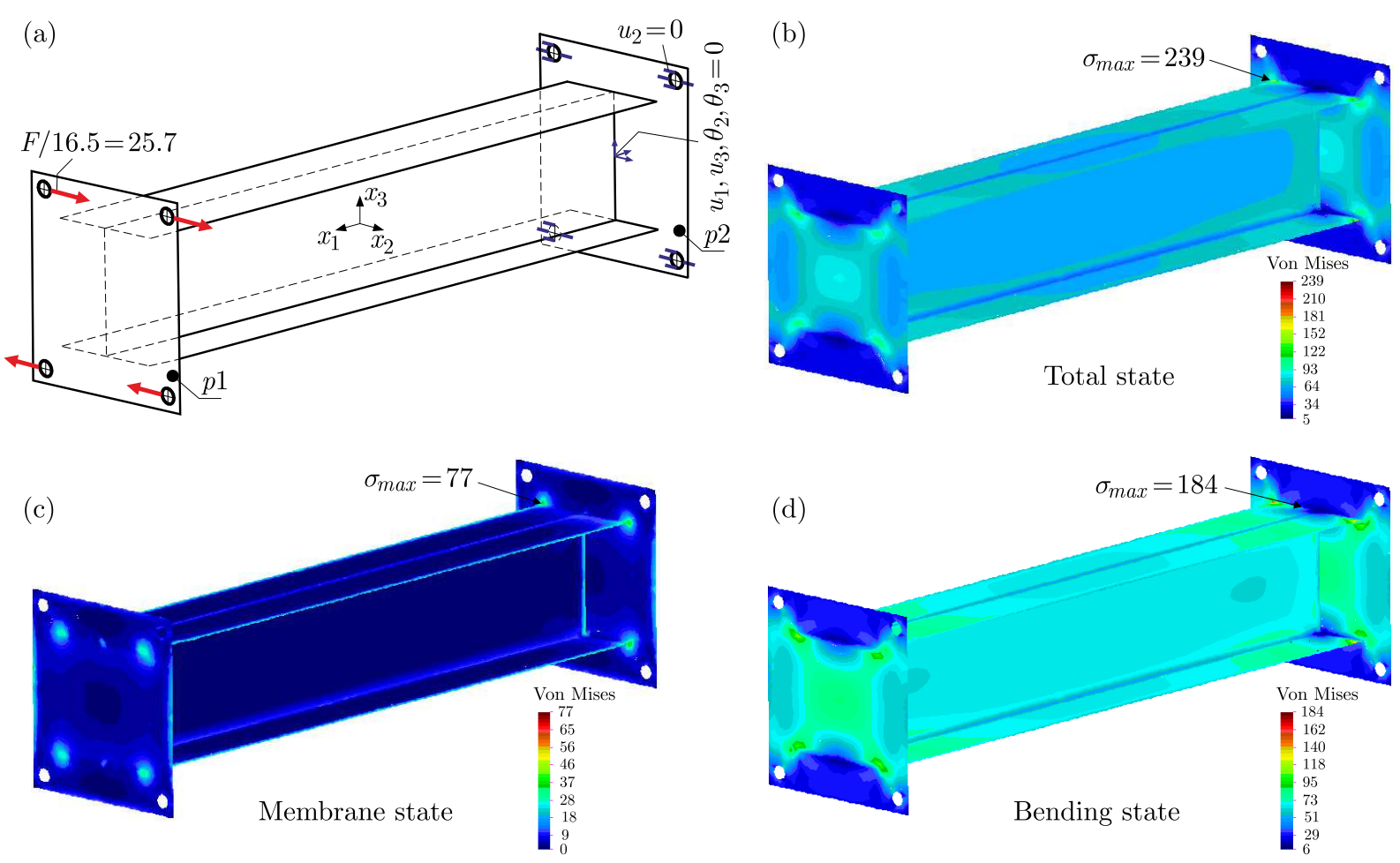

Fig. 7. The adopted boundary conditions and distribution of equivalent stresses calculated according to Huber-Mises-Hencky criterion for the model of regular double-tee section

the membrane state $(184 / 77 \approx 2.4)$, and the distribution of the total stress corresponds approximately to the distribution of the membrane state (Figs. $7 \mathrm{~b}$ and $7 \mathrm{~d}$ );

Rigidity of this model is: $\kappa \approx 6.2 \mathrm{Nm} / \mathrm{deg}$ and is as much as 51-times $(318 / 6.2 \approx 51)$ less comparing to rigidity of the model $Z 2 x 94$.

The above presentation of the equivalent stresses distribution shows how radically one may improve load-carrying properties and benefit from SADSF method application.

\section{Conclusions}

The application of the SADSF method, which does not make use of iterative procedures, can be successfully utilized while designing thin-walled structures wherein even a minor change of structural parameters may cause radical changes to load-carrying properties.

It may be applied already at the initial stage of designing, where only boundary conditions are known. At this stage, major load-carrying properties of thin-walled systems are determined.

The package of the application version of SADSFaM is not limited by complexity of a designed structure, it is easy to use and can be used by any engineer who knows the basics of statics. This means that it can be widely used in many practical applications.

As presented in the paper, having the given boundary conditions, sometimes one may construct a few solutions. This ambiguity can be used to search for such solutions which satisfy additional criteria, covering e.g. technological or strength limitations.

The designed structures do not include cardinal errors. Their structure configurations are adopted to transfer the assumed load, and one may expect in advance good load-carrying properties, radically better comparing to these systems designed using intuitive method. 
Current capabilities of the software in the application version of SADSF method as well as good strength properties of the designed structures show that this method is more and more popular among engineers.

\section{References}

1. Bendsoe M.P., Sigmund O., 2003, Topology Optimization: Theory, Methods, and Applications, Springer

2. Bodaszewski W., 1994, The investigation of the models of structural joint shells shaped from the condition of equalized effort in limit state (in Polish), XVI Sympozjum Mechaniki Eksperymentalnej Ciała Statego

3. Bodaszewski W., 2004, 2005, Algorithms of the method of statically admissible discontinuous stress fields (SADSF), Engineering Transactions, 52, 3, 175-193, 2004, 52, 4, 281-302, 2004, 53, 1, 15-30, 2005, 53, 2, 119-131, 2005

4. Bodaszewski W., 2013, Statical Analyses and Shaping of Complex Thin-Walled Structures (in Polish), BEL Studio, Warszawa

5. Bodaszewski W., Szczepiński W., 2005, Shaping Structure Elements by the Method of Discontinuous Stress Fields (in Polish), BEL Studio, PWN, Warszawa

6. Dietrich L., Miastkowski J., Szczepiński W., 1970, Load-Carrying Capacity of Structural Elements (in Polish), PWN, Warszawa

7. Frąckiewicz H., Szczepiński W., Tereszkowski Z., Szlagowski J., Bodaszewski W., Trela S., Barchan A., 1985, Joints and Structural Connections (in Polish), WNT, Warszawa

8. Huang X., Xie Y.M., 2010, Evolutionary Topology Optimization of Continuum Structures, Methods and Applications, Willey

9. Kapkowski J., Stupnicki J., 1973, Experimental investigations of machine elements designed by the method of load carrying capacity (in Polish), Rozprawy Inżynierskie, 21, 161-173

10. Markiewicz I., 2007, Analysis of elastic effort fields in truck frame designed by the SADSF method, Eksploatacja i Niezawodność - Maintenance and Reliability, 2, 34, 22-27

11. Markiewicz I., 2013, Investigating the Behaviour of Structures Designed with the SADSF Method (in Polish), Monografie, Studia, Rozprawy M47, Wydawnictwo Politechniki Świętokrzyskiej, Kielce

12. Mróz Z., Bojczuk D., 2003, Finite topology variations in optimal design of structures, Structural and Multidisciplinary Optimization, 25, 3, 153-173

13. SzcZePiński W., 1968, Plastic Design of Machine Parts (in Polish), PWN, Warszawa

14. Szczepiński W., Szlagowski J., 1990, Plastic Design of Complex Shape Structures, Ellis Horwood \& PWN, Warszawa-Chichester

15. SzLAGOWSKI J., 1990, Methodology of strength design of structural elements according to the limit load carrying capacity criterion (in Polish), IFTR Reports, 25

16. ZowCZAK W., 2004, Strength Design by Means of Slip-Line Method (in Polish), Monografie, Studia, Rozprawy M-41, Wydawnictwo Politechniki Świętokrzyskiej, Kielce 